\title{
Poisoning in Children: Utpal Kant Singh, FC Layland, Rajniti Prasad, Shivani Singh (eds)
}

\author{
Published by Jaypee Brothers Medical Publishers (P) Ltd, India, ISBN: 978-93-5025-773-9, \\ Fourth Edition: 2013
}

Poisoning in children is a common and preventable cause of morbidity and mortality. Our environment is still not childsafe and the medications are not dispensed in a child-safe manner. In sharp contrast to developed countries, where majority of poisonings are due to common household products, $40 \%$ patients do not develop symptoms, and upto three fourths of the symptomatic patients are discharged after a brief period of observation in the emergency ward, most of the patients in our country require hospitalization because of severe symptoms related to dangerous nature of toxins ingested. In such a scenario, it is important to promptly manage the numerous cases of poisoning in children. A reliable and handy reference for the same is desirable for achieving this goal.

The fourth edition of "Poisoning in Children" has now been published and includes many new chapters which readers will find useful. The authors have given a comprehensive general management plan in the initial chapters of the book which would help in initiation of management of the acutely sick child even if nature of the poisoning is unknown. The book discusses a wide spectrum of household and outdoor poisonings which are commonly observed in our geographical area. The book gives a brief overview of the relevant pathophysiology followed by point-wise listing of the clinical manifestations; this improves the readability of the book and the students will find this particularly useful. Listing of the key points in each chapter is of immense value.

There is scope for addition of some other relevant poisonings/toxicities like beta-blockers, digoxin, antiepileptics (phenytoin), etc. which are commonly prescribed medications and may be accidentally consumed by young children in the family. Arrangement of chapters in an alphabetical order may add to the utility of this resource.

This practical handbook has been written in a simple and reader friendly language with appropriate use of tables. This resource will be of much use not only for the undergraduate and postgraduate students but also for pediatricians and physicians looking after children.

D. Raj · R. Lodha $(\bowtie)$

Department of Pediatrics,

All India Institute of Medical Sciences,

Ansari Nagar,

New Delhi 110029,

India

e-mail: rakesh_lodha@hotmail.com 